\title{
An analysis of the learning community in the autonomous learning environment
}

\author{
Maohua SUN \\ Foreign Language Department, Dalian Jiao Tong University, Liaoning Province, China \\ summer_817@163.com
}

Keywords: learning community; autonomous learning; university teaching

\begin{abstract}
In recent years, learning community has been widely explored in the field of teacher development. It is a learning organization based on voluntary premise, shared vision and participation, dialogue and cooperation. Research shows that this kind of organization helps to promote the self-development of teachers [1]. It is based on this background that learning community mode promotes autonomous learning of college students. Promoting autonomous learning of college students is the key to the improvement of the quality of teaching and the development of the connotation. The study found that the learning community model can effectively promote college students' autonomous learning. The learning community model is of great significance for improving the learning effect of college students, improving the quality of the classroom teaching and enriching the methods of college teaching.
\end{abstract}

\section{学习共同体在自主学习环境中的探析 \\ 孙茂华}

大连交通大学, 大连, 辽宁省, 中国

summer_817@163.com

关键词：学习共同体; 自主学习; 大学教学

中文摘要. 近年来, 学习共同体在教师发展领域被广泛探讨, 它是一种以自愿为前提, 以共 同愿景为纽带, 以参与、对话和合作为核心的学习型组织。研究表明, 这种组织有助于促进 教师自主发展 [1]。以学习共同体模式促进大学生的自主学习, 正是基于这样的背景。促进 大学生自主学习是高校提升教学质量, 向内涵式发展的关键。研究发现, 学习共同体模式能 有效促进大学生自主学习。学习共同体模式对提高大学生课程学习效果、提升大学课堂教学 质量和丰富大学教学方法具有重要意义。

\section{1. 学习共同体与自主学习概述}

共同体一词, 作为社会学概念进入到学科领域可追溯到1887 年滕尼斯 (Ferdinad Tonnies) 的Gemeinschaft und Gesel lschaft 一书, 是指任何基于协作关系的有机组织形式 [2]。马克 斯 - 韦伯 (Max Weber) 对共同体和社会进行了区分, 他认为, 如果行为取向的基础是参与者 主观感受到同属于一个整体, 那这种社会关系称为 “共同体” ; 如果行为取向的基础是理性 驱动的利益平衡, 那这种社会关系就称为 “社会” [3]。1995 年, 博耶 (Ernest Boyer) 首 次提出了学习共同体的概念, 他指出, 学习共同体是朝着共同愿景努力的人所组成的组织, 共同体成员分享学习兴趣、共同寻找通向知识的旅程和理解世界运作的方式, 他们参与学习 并相互作用 [4]。我国学者也对学习共同体进行了界定。张建伟认为, 学习共同体是一个由 学习者及助学者 (教师、专家、辅导者等) 共同构成的团体, 他们之间经常交流, 分享学习资 
源, 完成一定的学习任务, 形成相互影响、相互促进的人际关系 [5]。薛焕玉认为, 学习共 同体是指一个由 “学习者与助学者共同构成的团体” ，他们拥有 “共同的目标” ，在共享的 环境中学习, 通过对话、交流和沟通来 “分享彼此的情感、体验和观念”, “共同完成一定 的学习任务” [6]。综合各学者的定义, 笔者认为学习共同体的要素可归纳为 “愿景” “参 与” “对话” “合作”和 “归属”。

自主学习（Self-Regulated Learning）是学习者系统地引导自己的思维、情感和行为, 使 它们指向目标实现的过程 [7]。它是学习者自我设立适宜的学习目标、制定合理的学业规 划、选择合适的学习内容、科学地进行时间管理、积极地寻求学习资源、主动地创设学习环 境并对学习进行有效监控和调整的过程。著名心理学家齐莫曼认为, 如果学生在学习动机、 方法、时间、环境、社会性和结果等方面均能由自己选择和控制, 那其学习就是充分自主的; 反之, 如果学生在这些方面均不能自己选择和控制, 则其学习就无自主性可言; 在实际学习 情境中，完全自主和完全不自主的学习都较少，多数学习介于两者之间 [8]。

\section{2. 英语自主学习的课堂环境因素}

课堂环境 (classroom climate) 是教师或学生对自己所在课堂的知觉或感受。最早提出环境 这一概念的是勒温 (Levin, 936), 他认为, 人类的行为是人与环境相互作用的结果, 即 $\mathrm{B}=\mathrm{f}(\mathrm{P}$, E)。之后, 穆斯 (Moos, 1979) 提出了环境三维度理论, 他从关系、个人发展以及系统维持 和变化三个维度描述了课堂环境的结构。关系维度涉及人际关系的本质和亲密度, 如教师帮 助并与学生交朋友的程度; 个人发展维度关注的是个人发展和自我增强的机会, 如学生合作 完成学习任务; 系统维持和变化维度评价的是环境的有序性程度以及对变化作出反应的程度, 如教师公平对待学生的程度等。20世纪80年代以后, 以Fraser 为代表的学者运用课堂环境测 量工具, 从课堂环境感知的角度对课堂环境与学生认知和情感成果之间的关系等进行了研究 和探讨。近年来, 国内对课堂环境的研究逐渐从介绍、评介西方课堂环境研究成果发展到对 中国教育背景下课堂环境的概念、特征、内容构成等的研究。范春林等认为, 课堂环境是指 影响教学活动开展和教学效果的, 并存在于课堂教学中的各种物理、社会及心理因素的总和, 既包括课堂物理环境, 也包括社会心理环境。本文的课堂环境主要是指课堂中的社会心理环 境, 也就是课堂中的师生关系和生生关系以及教师与学生的个性特征和心理状态等。英语自 主学习的课堂环境就是指有利于学生自主学习的师生课堂状态的总和, 包括良好的师生关系 和同伴关系、积极的师生课堂互动、明确的目标定向、科学的课堂组织方式和积极稳定的师 生心理环境等。

\section{3. 学习共同体的创建与使用}

建构主义理论指导下的多媒体英语学习的主要模式是基于网络技术的自主性学习以及协 作式学习, 所以网络多媒体的使用将会是学习共同体的主要途径。

\section{1 学习共同体的主体及辅助性软件}

\section{1 .1 网络教学平台}

广义的网络平台包括支持网络教学的硬件设施和支持网络教学的软件系统。笔者所在的 学校为保证教师与学生之间时刻保持畅通的交流和联系、教学材料、课程通知、课程作业的 上传下达, 设立了属于本校学生的网络教学平台。在此平台中教师可进行集中发起教学的问 卷调查, 分享教学博客, 并可以通过邮箱和论坛与学生进行交流。除此之外, 网络教学平台 还设置了教学大纲、教学日历、教学材料、课程通知、答疑讨论、课程问卷、学习笔记、研 究型教学、课程作业、试题试卷库、在线测试、学习记录等多个板块配合教学, 非常方便地 进行学习共同体的资源配置, 将现有的学习平台发挥出最大的效果。 


\section{1.2 辅助性软件}

辅助性软件具体是指当下较为流行的QQ和微信。它们都属于社会性软件中即时通讯软件 （IM）。这些软件在很大程度上弥补了网络教学平台不能随时随地收发信息、资料的不足。 此外，微信独有的公众号推送也将是一个学习者之间分享学习经验和资源的好平台。

\section{2 创建学习共同体}

\subsubsection{QQ群的创建}

为配合网络教学平台的使用, 课题小组建立了QQ群, 然后将学习者和助学者都添加到群 里, 并改正了群名片, 以明确助学者的身份。同时完善通知栏、课程组织、日历、文件与资 源管理、内容讨论区、管理讨论区、成员列表、电子邮件、意见和建议反馈区、学习情况统 计、学生个人主页等多项常用学习共同体工具, 帮助学习者进行更为便捷有效的学习和成员 间的资源交流。此外, 进一步明确对群组中闲聊天、发送与学习无关的信息、不按时完成任 务的成员的惩罚措施, 使QQ群的内部环境变得更加规范有序。

\section{2 .2 微信群聊、公众号的创建}

微信是近几年来迅速流行的一种交流软件, 比起QQ它的操作简单方便, 且更适合进行语音 的发送。在雅思的考试中, 口语也十分重要, 因此微信是提交口语作业的最好选择。群聊的 创建方式与 $Q Q$ 群的创建别无二致, 不多做赘述。公众号的每日推送则作为日常学习者的任务, 将落实到学习共同体的每一个人身上, 作为学习经验的分享。在每日推送前由指导教师审核, 以确保推送内容的有效性。

\section{3 使用学习共同体}

在研究中，学习者每日配合网络平台上发布的教学大纲和教学日历进行预习, 课上听教 师正常的讲课, 课下通过QQ群每周话题讨论、微信公众平台经验分享等方式进行学习者之间 的交流。学习者将在课堂的学习过程中或自主学习过程中遇到的问题或需求做出总结。学习 共同体中的助学教师会发布与问题和需求相关的学习资料或学习方法, 使问题得到最快的解 决。同时, 学习者就在学习共同体中总结到的问题, 与其授课教师进行了反向沟通, 主动影 响了老师的授课方式和授课内容, 从而进一步完善了自身的学习。具体使用内容和结果如下: 3. 3.1 听力方面

教师在课后上传与课堂教学相关的教学材料到网络教学平台上, 学习者在下载后必须在规 定时间内完成对音频英语内容由音到文的转换, 并将文档材料传送到QQ群中。教师会根据每 个学习者提交上来的作业分析制定今后的训练方向, 从而改善学习者如记忆障碍、词汇量不 够、对文章相关文化背景不通等方面的问题。

\subsection{2 口语方面}

雅思口语考试由三个部分组成, 即简介及问答、个人陈述和双向讨论。这一训练除了在课 堂上进行, 还可以通过微信群完成。在微信群中, 教师与学习者之间、学习者与学习者之间 模拟了考试的对话形式, 而后在每周的讨论时间分析学习共同体中每位成员口语上出现的技 巧或语言方面的问题。这不仅使学习者适应了雅思口语考试的流程和模式, 更使得学习者更 为深刻地了解到自身的不足, 并在这个大家共同改进自身的大环境下, 树立了提高自己的自 信心。

\subsection{3 阅读与写作方面}

限时, 准确获取信息, 迅速罗列观点是阅读与写作的训练重点。从短小的文章到长篇, 学习者不仅利用阅读时总结上传到QQ群中的文化知识和词汇辅助提高写作能力, 也通过写作 中观点延伸的训练帮助了雅思阅读中段落层次的划分, 形成双向性的提高。此外, 微信公众 平台的推送也在看似不经意间对学习者关于英语的文化背景产生了潜移默化的影响。 


\section{3.4 情绪方面}

在学习共同体开始进行之前, 不少学习者表示处于一种学而无法的焦躁状态中。在共同 体中, 学习者们聚集在一起相互交流学习经验, 共同协作完成任务和训练, 加上时常会有专 业教师的指导和沟通, 其学习态度和学习热情得到了前所未有的 “共振效应”。可以说与志 同道合的伙伴们并肩作战不仅为他们带来了能力上的提升，更激发了他们精神上的动力。

\section{4. 总结}

实践证明, 学习共同体模式对促进大学生自主学习具有积极成效, 这一模式可以促使教 学从 “以教师为中心” 向 “以学生为中心” 的转变, 让课堂真正成为学生自主成长的地方。 学习共同体模式对提高大学生课程学习效果、提升大学课堂教学质量和丰富大学教学方法有 诸多裨益。首先, 课程是师生的共同创造。学习目标在师生互动中生成, 学习资源由师生共 同获取, 教学组织由师生共同完成, 这能促使学生最大限度地参与学习并取得好的学习成效。 其次, 课堂是有温度的。师生在教学活动中感受到相互的尊重和信任, 在双向的努力中形成 对团体的归属, 这使得课堂教学充满生命力, 有助于课堂教学质量提升。最后, 鼓励朋辈学 习的教学。无顾虑的交流和自在的朋辈关系, 使学生获得自由的成长; 朋辈学习是大学教学 中最富生机的生长点。学习共同体的实践方兴未艾, 未来教育者可在更多层面利用这类组织 的优势探索大学教学质量的提升。

\section{References}

[1] Zhan Zehui, Li Xiaohua. The construction of American University Teachers' learning community -- dialogue, Professor Milton Kors, Professor of teaching promotion center, University of Miami [J]. Audiovisual education in China, 2009 (10).

[2] Louisa Ferdinand Tonnies. Community and society -- the basic concept of M pure sociology. Lin Rongyuan, transl. Beijing: commercial press gallery, 1999:71-72.

[3] [beauty] Max Webb. The basic concept of sociology [M]. Gu Zhonghua, Guangxi: Guangxi Normal University press, 2005:54.

[4] Boyer E. A Basic School: A Community for Learning [R].Princeton.

[5] Zhang Jianwei, Sun Yanqing. Constructivist learning -- an integrated exploration of learning science [M]. Shanghai: Shanghai Education Press, 2005:98.

[6] Xue Yuhuan. A preliminary study of the theory and practice of the learning community [J]. Journal of China University of Geosciences: Social Science Edition, 2007 (1).

[7] Schunk D H, Zimmerman B J. Self-regulation Learning andPerformanc [M].Lawrence Erillbaum, 8 .

[8] Zimmerman B J. Dimension of Academic Self-regulation [M] //Schunk D H, the D, J., 8.

[9] Zhang Jianwei, Sun Yanqing. Constructivist learning -- an integrated exploration of learning science [M]. Shanghai: Shanghai Education Press, 2005.

[10] Guo Yongzhi. Research on the construction of College English learning model based on the learning community theory $[\mathrm{J}]$. Journal of the school of education administration in Liaoning, 2010, (10): 92-93.

[11] Wu Tongjun. Building a college English learning community in the network virtual environment [J]. Journal of Capital Normal University (SOCIAL SCIENCE EDITION), 2010, (Supplement): 65-67. 
[12] $\mathrm{Yu}$. Educational technology and education to promote two-way integration -- "education informatization development planning ten years (2011-2020 years)" on J. Chinese audio-visual education, 2012 (5): 5-14. 